\title{
Hermite-Hadamard-type inequalities for conformable integrals
}

\author{
Martin Bohner*1 (D), Artion Kashuri² (D), \\ Pshtiwan Othman Mohammed ${ }^{3}$ (D), Juan E. Nápoles Valdés ${ }^{4}$ (D) \\ ${ }^{1}$ Missouri S8T, Rolla, MO 65409, USA \\ ${ }^{2}$ University Ismail Qemali, Vlora, Albania \\ ${ }^{3}$ University of Sulaimani, Sulaimani, Iraq \\ ${ }^{4}$ Universidad Nacional del Nordeste, Corrientes 3400, Argentina
}

\begin{abstract}
In this study, some inequalities of Hermite-Hadamard type for integrals arising in conformable fractional calculus are presented. In fact, the obtained inequalities are not only valid for those integrals arising in conformable fractional calculus, but for more general integrals as well. Numerous known versions are recovered as special cases. We also illustrate our findings via applications to modified Bessel functions, special means, and midpoint approximations.
\end{abstract}

Mathematics Subject Classification (2020). 26D07, 26D15, 26D10, 26A33

Keywords. Hermite-Hadamard inequality, convex function, conformable integral

\section{Introduction}

In [1], a so-called conformable fractional integral of the form

$$
\int_{\rho_{1}}^{\rho_{2}} f(x) \mathrm{d}_{\omega} x=\int_{\rho_{1}}^{\rho_{2}} x^{\omega-1} f(x) \mathrm{d} x
$$

(if it exists and is finite) was introduced for $\omega \in(0,1]$. In view of recent results in the theories of differential, integral, and fractional differential equations, it becomes clear that certain integral inequalities are very useful in determining bounds of unknown functions; see, e.g., $[2,6-9,11,14,15,19,25,29]$. Also, there are various integral inequalities in the literature, and research in this area is very active. One main inequality is the HermiteHadamard integral inequality, due to Hadamard (1893), which says that for convex functions $f$, we have

$$
f\left(\frac{\rho_{1}+\rho_{2}}{2}\right) \leq \frac{1}{\rho_{2}-\rho_{1}} \int_{\rho_{1}}^{\rho_{2}} f(x) \mathrm{d} x \leq \frac{f\left(\rho_{1}\right)+f\left(\rho_{2}\right)}{2} .
$$

Inequality (1.2) has been extended and applied to time scales $[4,5]$ and to many models of fractional calculus, such as Riemann-Liouville [18,21,26], $\psi$-Riemann-Liouville [17],

\footnotetext{
*Corresponding Author.

Email addresses: bohner@mst.edu (M. Bohner), artionkashuri@gmail.com (A. Kashuri), pshtiwansangawi@gmail.com (P.O. Mohammed), profjnapoles@gmail.com (J.E. Nápoles Valdés)

Received: 31.05.2021; Accepted: 02.12.2021
} 
conformable fractional [20], generalized fractional [22,24], time scales fractional [3, 28], and tempered fractional [23].

In this article, after proving some auxiliary results in Section 2, we devote Section 3 to obtain three distinct inequalities of Hermite-Hadamard type for integrals of the form (1.1). In each case, by choosing $\omega=1$, we recover known results from the literature. In Section 4 , we apply our results to integer values of $\omega$, and in Section 4 , we demonstrate the usefulness of our inequalities by offering three different applications. We emphasize that our Hermite-Hadamard inequalities for conformable integrals (1.1) collapse to known Hermite-Hadamard inequalities for integrals when $\omega=1$.

\section{Auxiliary results}

At first, we recall the definition of convexity. We say that $f: I \rightarrow \mathbb{R}$ is convex on the interval $I$ if

$$
f(\eta x+(1-\eta) y) \leq \eta f(x)+(1-\eta) f(y) \quad \text { for all } \quad x, y \in I \quad \text { and } \quad \eta \in[0,1] .
$$

In the first auxiliary result, we collect some formulas that will be needed in the proofs of our main results.

Lemma 2.1. Let $\omega>0$ and $z_{1} \neq z_{2}$. Denote

$$
\begin{gathered}
z_{*}:=\frac{z_{1}+z_{2}}{2}, \\
G\left(z_{1}, z_{2}\right):=\int_{z_{1}}^{z_{*}}\left(x^{\omega}-z_{1}^{\omega}\right) \mathrm{d} x, \\
F_{1}\left(z_{1}, z_{2}\right):=\frac{1}{z_{2}-z_{1}} \int_{z_{1}}^{z_{*}}\left(x^{\omega}-z_{1}^{\omega}\right)\left(x-z_{1}\right) \mathrm{d} x, \\
F_{2}\left(z_{1}, z_{2}\right):=\frac{1}{z_{2}-z_{1}} \int_{z_{1}}^{z_{*}}\left(x^{\omega}-z_{1}^{\omega}\right)\left(z_{2}-x\right) \mathrm{d} x .
\end{gathered}
$$

Then we have

$$
\begin{gathered}
G\left(z_{1}, z_{2}\right)=\frac{z_{*}^{\omega+1}-z_{1}^{\omega+1}}{\omega+1}-\frac{z_{1}^{\omega}\left(z_{2}-z_{1}\right)}{2}, \\
\int_{z_{*}}^{z_{2}}\left(z_{2}^{\omega}-x^{\omega}\right) \mathrm{d} x=G\left(z_{2}, z_{1}\right), \\
F_{1}\left(z_{1}, z_{2}\right)=\frac{z_{1}^{\omega+2}-z_{*}^{\omega+2}}{(\omega+1)(\omega+2)\left(z_{2}-z_{1}\right)}+\frac{z_{*}^{\omega+1}}{2(\omega+1)}-z_{1}^{\omega} \frac{z_{2}-z_{1}}{8}, \\
F_{2}\left(z_{1}, z_{2}\right)=\frac{z_{*}^{\omega+2}-z_{1}^{\omega+2}}{(\omega+1)(\omega+2)\left(z_{2}-z_{1}\right)}+\frac{\frac{z_{*}^{\omega+1}}{2}-z_{1}^{\omega+1}}{\omega+1}-3 z_{1}^{\omega} \frac{z_{2}-z_{1}}{8}, \\
\frac{1}{z_{2}-z_{1}} \int_{z_{*}}^{z_{2}}\left(z_{2}^{\omega}-x^{\omega}\right)\left(x-z_{1}\right) \mathrm{d} x=F_{2}\left(z_{2}, z_{1}\right), \\
\frac{1}{z_{2}-z_{1}} \int_{z_{*}}^{z_{2}}\left(z_{2}^{\omega}-x^{\omega}\right)\left(z_{2}-x\right) \mathrm{d} x=F_{1}\left(z_{2}, z_{1}\right) .
\end{gathered}
$$

Proof. A simple integration verifies (2.1). The definition of $G$ shows (2.2). Now we verify the second equal sign in (2.3). We use (2.1) three times (with replacements of $\omega$ by $\omega+1, \omega, 1)$ as well as twice the formula

$$
z_{*}-z_{1}=\frac{z_{2}-z_{1}}{2}
$$

to find

$$
F_{1}\left(z_{1}, z_{2}\right)=\frac{1}{z_{2}-z_{1}} \int_{z_{1}}^{z_{*}}\left[x^{\omega+1}-z_{1}^{\omega+1}-z_{1}\left(x^{\omega}-z_{1}^{\omega}\right)-z_{1}^{\omega}\left(x-z_{1}\right)\right] \mathrm{d} x
$$




$$
\begin{aligned}
& =\frac{z_{*}^{\omega+2}-z_{1}^{\omega+2}}{(\omega+2)\left(z_{2}-z_{1}\right)}-\frac{z_{1}^{\omega+1}}{2}-z_{1}\left(\frac{z_{*}^{\omega+1}-z_{1}^{\omega+1}}{(\omega+1)\left(z_{2}-z_{1}\right)}-\frac{z_{1}^{\omega}}{2}\right)-z_{1}^{\omega}\left(\frac{z_{*}^{2}-z_{1}^{2}}{2\left(z_{2}-z_{1}\right)}-\frac{z_{1}}{2}\right) \\
& =\frac{z_{1}^{\omega+2}-z_{*}^{\omega+2}+(\omega+2)\left(z_{*}^{\omega+2}-z_{1}^{\omega+2}\right)}{(\omega+1)(\omega+2)\left(z_{2}-z_{1}\right)}+\frac{z_{1}^{\omega+2}-z_{1} z_{*}^{\omega+1}}{(\omega+1)\left(z_{2}-z_{1}\right)}-z_{1}^{\omega}\left(\frac{z_{*}+z_{1}}{4}-\frac{z_{1}}{2}\right) .
\end{aligned}
$$

Since $F_{1}+F_{2}=G,(2.4)$ is just a simple calculation using (2.1) and (2.3). Finally, (2.5) and (2.6) follow from the definitions of $F_{2}$ and $F_{1}$, respectively.

Remark 2.2. If we let $\omega=1$ in Lemma 2.1 and use (2.7) several times, then we have

$$
\begin{aligned}
& G\left(z_{1}, z_{2}\right)=\int_{z_{1}}^{z_{*}}\left(x-z_{1}\right) \mathrm{d} x=\frac{\left(z_{*}-z_{1}\right)^{2}}{2}=\frac{\left(z_{2}-z_{1}\right)^{2}}{8} \\
& F_{1}\left(z_{1}, z_{2}\right)=\frac{1}{z_{2}-z_{1}} \int_{z_{1}}^{z_{*}}\left(x-z_{1}\right)^{2} \mathrm{~d} x=\frac{\left(z_{*}-z_{1}\right)^{3}}{3\left(z_{2}-z_{1}\right)}=\frac{\left(z_{2}-z_{1}\right)^{2}}{24}, \\
& F_{2}\left(z_{1}, z_{2}\right)=G\left(z_{1}, z_{2}\right)-F_{1}\left(z_{1}, z_{2}\right)=\frac{\left(z_{2}-z_{1}\right)^{2}}{12} .
\end{aligned}
$$

Now, the following lemma is a key to obtain our main results. In the sequel, we assume $\left(\mathrm{H}_{\omega}\right) \omega>0,0 \leq \rho_{1}<\rho_{2}$, and $f:\left[\rho_{1}, \rho_{2}\right] \rightarrow \mathbb{R}$ is such that

$$
\wp_{1}:=\int_{\rho_{1}}^{\rho_{*}}\left(x^{\omega}-\rho_{1}^{\omega}\right) f^{\prime}(x) \mathrm{d} x \quad \text { and } \quad \wp_{2}:=\int_{\rho_{*}}^{\rho_{2}}\left(\rho_{2}^{\omega}-x^{\omega}\right) f^{\prime}(x) \mathrm{d} x
$$

are well defined. Recall $\rho_{*}=\left(\rho_{1}+\rho_{2}\right) / 2$.

Lemma 2.3. If $\left(H_{\omega}\right)$ holds, then

$$
\frac{\omega}{\rho_{2}^{\omega}-\rho_{1}^{\omega}} \int_{\rho_{1}}^{\rho_{2}} f(x) \mathrm{d}_{\omega} x-f\left(\rho_{*}\right)=\frac{\wp_{2}-\wp_{1}}{\rho_{2}^{\omega}-\rho_{1}^{\omega}} .
$$

Proof. Using integration by parts and (1.1), we get

$$
\begin{aligned}
\wp_{1} & =\int_{\rho_{1}}^{\rho_{*}}\left(x^{\omega}-\rho_{1}^{\omega}\right) f^{\prime}(x) \mathrm{d} x \\
& =\left.\left(x^{\omega}-\rho_{1}^{\omega}\right) f(x)\right|_{\rho_{1}} ^{\rho_{*}}-\int_{\rho_{1}}^{\rho_{*}} \omega x^{\omega-1} f(x) \mathrm{d} x \\
& =\left(\rho_{*}^{\omega}-\rho_{1}^{\omega}\right) f\left(\rho_{*}\right)-\omega \int_{\rho_{1}}^{\rho_{*}} f(x) \mathrm{d}_{\omega} x .
\end{aligned}
$$

Similarly,

$$
\begin{aligned}
\wp_{2} & =\int_{\rho_{*}}^{\rho_{2}}\left(\rho_{2}^{\omega}-x^{\omega}\right) f^{\prime}(x) \mathrm{d} x \\
& =\left.\left(\rho_{2}^{\omega}-x^{\omega}\right) f(x)\right|_{\rho_{*}} ^{\rho_{2}}+\int_{\rho_{*}}^{\rho_{2}} \omega x^{\omega-1} f(x) \mathrm{d} x \\
& =\omega \int_{\rho_{*}}^{\rho_{2}} f(x) \mathrm{d} \omega x-\left(\rho_{2}^{\omega}-\rho_{*}^{\omega}\right) f\left(\rho_{*}\right) .
\end{aligned}
$$

Altogether, we have

$$
\wp_{2}-\wp_{1}=\omega \int_{\rho_{1}}^{\rho_{2}} f(x) \mathrm{d}_{\omega} x-\left(\rho_{2}^{\omega}-\rho_{1}^{\omega}\right) f\left(\rho_{*}\right),
$$

from which we obtain the desired identity (2.8). This completes the proof.

Remark 2.4. If we take absolute values in (2.8), then we obtain the inequality

$$
\left|\frac{\omega}{\rho_{2}^{\omega}-\rho_{1}^{\omega}} \int_{\rho_{1}}^{\rho_{2}} f(x) \mathrm{d}_{\omega} x-f\left(\rho_{*}\right)\right| \leq \frac{\left|\wp_{1}\right|+\left|\wp_{2}\right|}{\rho_{2}^{\omega}-\rho_{1}^{\omega}} .
$$




\section{Main results}

Now we present the three main results of this paper.

Theorem 3.1. If $\left(H_{\omega}\right)$ holds and $\left|f^{\prime}\right|$ is convex, then

where

$$
\left|\frac{\omega}{\rho_{2}^{\omega}-\rho_{1}^{\omega}} \int_{\rho_{1}}^{\rho_{2}} f(x) \mathrm{d}_{\omega} x-f\left(\rho_{*}\right)\right| \leq \frac{F\left(\rho_{1}, \rho_{2}\right)\left|f^{\prime}\left(\rho_{1}\right)\right|+F\left(\rho_{2}, \rho_{1}\right)\left|f^{\prime}\left(\rho_{2}\right)\right|}{\rho_{2}^{\omega}-\rho_{1}^{\omega}},
$$

$$
F\left(z_{1}, z_{2}\right):=\frac{2 z_{*}^{\omega+2}-z_{1}^{\omega+2}-z_{2}^{\omega+2}}{(\omega+1)(\omega+2)\left(z_{2}-z_{1}\right)}+\frac{z_{*}^{\omega+1}-z_{1}^{\omega+1}}{\omega+1}+\left(z_{2}^{\omega}-3 z_{1}^{\omega}\right) \frac{z_{2}-z_{1}}{8}, \quad z_{1} \neq z_{2} .
$$

Proof. By the convexity of $\left|f^{\prime}\right|$, we get

$$
\left|f^{\prime}(x)\right|=\left|f^{\prime}\left(\frac{\rho_{2}-x}{\rho_{2}-\rho_{1}} \rho_{1}+\frac{x-\rho_{1}}{\rho_{2}-\rho_{1}} \rho_{2}\right)\right| \leq \frac{\rho_{2}-x}{\rho_{2}-\rho_{1}}\left|f^{\prime}\left(\rho_{1}\right)\right|+\frac{x-\rho_{1}}{\rho_{2}-\rho_{1}}\left|f^{\prime}\left(\rho_{2}\right)\right|,
$$

and thus

$$
\begin{aligned}
\left|\wp_{1}\right| & \leq \int_{\rho_{1}}^{\rho_{*}}\left(x^{\omega}-\rho_{1}^{\omega}\right)\left|f^{\prime}(x)\right| \mathrm{d} x \\
& \leq \int_{\rho_{1}}^{\rho_{*}}\left(x^{\omega}-\rho_{1}^{\omega}\right)\left[\frac{\rho_{2}-x}{\rho_{2}-\rho_{1}}\left|f^{\prime}\left(\rho_{1}\right)\right|+\frac{x-\rho_{1}}{\rho_{2}-\rho_{1}}\left|f^{\prime}\left(\rho_{2}\right)\right|\right] \mathrm{d} x \\
& =F_{2}\left(\rho_{1}, \rho_{2}\right)\left|f^{\prime}\left(\rho_{1}\right)\right|+F_{1}\left(\rho_{1}, \rho_{2}\right)\left|f^{\prime}\left(\rho_{2}\right)\right| .
\end{aligned}
$$

Similarly,

$$
\begin{aligned}
\left|\wp_{2}\right| & \leq \int_{\rho_{*}}^{\rho_{2}}\left(\rho_{2}^{\omega}-x^{\omega}\right)\left|f^{\prime}(x)\right| \mathrm{d} x \\
& \leq \int_{\rho_{*}}^{\rho_{2}}\left(\rho_{2}^{\omega}-x^{\omega}\right)\left[\frac{\rho_{2}-x}{\rho_{2}-\rho_{1}}\left|f^{\prime}\left(\rho_{1}\right)\right|+\frac{x-\rho_{1}}{\rho_{2}-\rho_{1}}\left|f^{\prime}\left(\rho_{2}\right)\right|\right] \mathrm{d} x \\
& =F_{1}\left(\rho_{2}, \rho_{1}\right)\left|f^{\prime}\left(\rho_{1}\right)\right|+F_{2}\left(\rho_{2}, \rho_{1}\right)\left|f^{\prime}\left(\rho_{2}\right)\right|,
\end{aligned}
$$

where we have also used (2.5) and (2.6). From (2.3) and (2.4), it is easy to see that

$$
F_{1}\left(z_{2}, z_{1}\right)+F_{2}\left(z_{1}, z_{2}\right)=F\left(z_{1}, z_{2}\right) .
$$

Employing now (2.9) completes the proof.

When we choose $\omega=1$ in Theorem 3.1, we get the following result.

Corollary 3.2. If $\left(H_{1}\right)$ holds and $\left|f^{\prime}\right|$ is convex, then we have

$$
\left|\frac{1}{\rho_{2}-\rho_{1}} \int_{\rho_{1}}^{\rho_{2}} f(x) \mathrm{d} x-f\left(\rho_{*}\right)\right| \leq \frac{\rho_{2}-\rho_{1}}{8}\left(\left|f^{\prime}\left(\rho_{1}\right)\right|+\left|f^{\prime}\left(\rho_{2}\right)\right|\right) .
$$

Proof. If $\omega=1$, then, due to (3.1) and Remark 2.2, we get

$$
F\left(z_{1}, z_{2}\right)=F_{1}\left(z_{2}, z_{1}\right)+F_{2}\left(z_{1}, z_{2}\right)=\frac{\left(z_{2}-z_{1}\right)^{2}}{24}+\frac{\left(z_{2}-z_{1}\right)^{2}}{12}=\frac{\left(z_{2}-z_{1}\right)^{2}}{8}
$$

and thus the statement follows from Theorem 3.1.

Remark 3.3. Note that Corollary 3.2 was proved first in [13, Theorem 2.1]. Hence, our Theorem 3.1 is a generalization of [13, Theorem 2.1].

Theorem 3.4. If $\left(H_{\omega}\right)$ holds and $\left|f^{\prime}\right|^{q}$ is convex for some $q \geq 1$, then

$$
\begin{aligned}
& \left|\frac{\omega}{\rho_{2}^{\omega}-\rho_{1}^{\omega}} \int_{\rho_{1}}^{\rho_{2}} f(x) \mathrm{d}_{\omega} x-f\left(\rho_{*}\right)\right| \\
& \quad \leq \frac{\left(G\left(\rho_{1}, \rho_{2}\right)\right)^{1-\frac{1}{q}}}{\rho_{2}^{\omega}-\rho_{1}^{\omega}}\left(F_{2}\left(\rho_{1}, \rho_{2}\right)\left|f^{\prime}\left(\rho_{1}\right)\right|^{q}+F_{1}\left(\rho_{1}, \rho_{2}\right)\left|f^{\prime}\left(\rho_{2}\right)\right|^{q}\right)^{\frac{1}{q}}
\end{aligned}
$$




$$
+\frac{\left(G\left(\rho_{2}, \rho_{1}\right)\right)^{1-\frac{1}{q}}}{\rho_{2}^{\omega}-\rho_{1}^{\omega}}\left(F_{1}\left(\rho_{2}, \rho_{1}\right)\left|f^{\prime}\left(\rho_{1}\right)\right|^{q}+F_{2}\left(\rho_{2}, \rho_{1}\right)\left|f^{\prime}\left(\rho_{2}\right)\right|^{q}\right)^{\frac{1}{q}}
$$

where $F_{1}, F_{2}$, and $G$ are given in Lemma 2.1.

Proof. By the convexity of $\left|f^{\prime}\right|^{q}$, we get

$$
\left|f^{\prime}(x)\right|^{q}=\left|f^{\prime}\left(\frac{\rho_{2}-x}{\rho_{2}-\rho_{1}} \rho_{1}+\frac{x-\rho_{1}}{\rho_{2}-\rho_{1}} \rho_{2}\right)\right|^{q} \leq \frac{\rho_{2}-x}{\rho_{2}-\rho_{1}}\left|f^{\prime}\left(\rho_{1}\right)\right|^{q}+\frac{x-\rho_{1}}{\rho_{2}-\rho_{1}}\left|f^{\prime}\left(\rho_{2}\right)\right|^{q},
$$

and thus, with the help of the power-mean inequality,

$$
\begin{aligned}
\left|\wp_{1}\right| & \leq \int_{\rho_{1}}^{\rho_{*}}\left(x^{\omega}-\rho_{1}^{\omega}\right)\left|f^{\prime}(x)\right| \mathrm{d} x \\
& \leq\left(\int_{\rho_{1}}^{\rho_{*}}\left(x^{\omega}-\rho_{1}^{\omega}\right) \mathrm{d} x\right)^{1-\frac{1}{q}}\left(\int_{\rho_{1}}^{\rho_{*}}\left(x^{\omega}-\rho_{1}^{\omega}\right)\left|f^{\prime}(x)\right|^{q} \mathrm{~d} x\right)^{\frac{1}{q}} \\
& =\left(G\left(\rho_{1}, \rho_{2}\right)\right)^{1-\frac{1}{q}}\left(\int_{\rho_{1}}^{\rho_{*}}\left(x^{\omega}-\rho_{1}^{\omega}\right)\left|f^{\prime}(x)\right|^{q} \mathrm{~d} x\right)^{\frac{1}{q}} \\
& \leq\left(G\left(\rho_{1}, \rho_{2}\right)\right)^{1-\frac{1}{q}}\left(F_{2}\left(\rho_{1}, \rho_{2}\right)\left|f^{\prime}\left(\rho_{1}\right)\right|^{q}+F_{1}\left(\rho_{1}, \rho_{2}\right)\left|f^{\prime}\left(\rho_{2}\right)\right|^{q}\right)^{\frac{1}{q}} .
\end{aligned}
$$

Similarly,

$$
\begin{aligned}
\left|\wp_{2}\right| & \leq \int_{\rho_{*}}^{\rho_{2}}\left(\rho_{2}^{\omega}-x^{\omega}\right)\left|f^{\prime}(x)\right| \mathrm{d} x \\
& \leq\left(\int_{\rho_{*}}^{\rho_{2}}\left(\rho_{2}^{\omega}-x^{\omega}\right) \mathrm{d} x\right)^{1-\frac{1}{q}}\left(\int_{\rho_{*}}^{\rho_{2}}\left(\rho_{2}^{\omega}-x^{\omega}\right)\left|f^{\prime}(x)\right|^{q} \mathrm{~d} x\right)^{\frac{1}{q}} \\
& =\left(G\left(\rho_{2}, \rho_{1}\right)\right)^{1-\frac{1}{q}}\left(\int_{\rho_{*}}^{\rho_{2}}\left(\rho_{2}^{\omega}-x^{\omega}\right)\left|f^{\prime}(x)\right|^{q} \mathrm{~d} x\right)^{\frac{1}{q}} \\
& \leq\left(G\left(\rho_{2}, \rho_{1}\right)\right)^{1-\frac{1}{q}}\left(F_{1}\left(\rho_{2}, \rho_{1}\right)\left|f^{\prime}\left(\rho_{1}\right)\right|^{q}+F_{2}\left(\rho_{2}, \rho_{1}\right)\left|f^{\prime}\left(\rho_{2}\right)\right|^{q}\right)^{\frac{1}{q}},
\end{aligned}
$$

where we have also used (2.2), (2.5), and (2.6). Employing now (2.9) completes the proof.

Remark 3.5. Theorem 3.4 with $q=1$ becomes Theorem 3.1.

When we choose $\omega=1$ in Theorem 3.4, we get the following result.

Corollary 3.6. If $\left(H_{1}\right)$ holds and $\left|f^{\prime}\right|^{q}$ is convex for some $q \geq 1$, then

$$
\begin{aligned}
\mid \frac{1}{\rho_{2}-\rho_{1}} \int_{\rho_{1}}^{\rho_{2}} f(x) \mathrm{d} x & -f\left(\rho_{*}\right) \mid \\
\leq & \frac{\rho_{2}-\rho_{1}}{8 \cdot 3^{\frac{1}{q}}}\left[\left(2\left|f^{\prime}\left(\rho_{1}\right)\right|^{q}+\left|f^{\prime}\left(\rho_{2}\right)\right|^{q}\right)^{\frac{1}{q}}+\left(\left|f^{\prime}\left(\rho_{1}\right)\right|^{q}+2\left|f^{\prime}\left(\rho_{2}\right)\right|^{q}\right)^{\frac{1}{q}}\right] .
\end{aligned}
$$

Proof. This follows directly from Theorem 3.4 and Remark 2.2.

Remark 3.7. Note that Corollary 3.6 was proved first in [10, Proof of Corollary 1]. Hence, our Theorem 3.4 is a generalization of [10, Corollary 1].

Theorem 3.8. If $\left(H_{\omega}\right)$ holds and $\left|f^{\prime}\right|^{q}$ is convex for some $q>1$, then

$$
\begin{array}{r}
\left|\frac{\omega}{\rho_{2}^{\omega}-\rho_{1}^{\omega}} \int_{\rho_{1}}^{\rho_{2}} f(x) \mathrm{d}_{\omega} x-f\left(\rho_{*}\right)\right| \leq \frac{\left(\rho_{2}-\rho_{1}\right)^{\frac{1}{q}}}{8^{\frac{1}{q}}\left(\rho_{2}^{\omega}-\rho_{1}^{\omega}\right)}\left\{\left(G_{p}\left(\rho_{1}, \rho_{2}\right)\right)^{\frac{1}{p}}\left(3\left|f^{\prime}\left(\rho_{1}\right)\right|^{q}+\left|f^{\prime}\left(\rho_{2}\right)\right|^{q}\right)^{\frac{1}{q}}\right. \\
\left.+\left(G_{p}\left(\rho_{2}, \rho_{1}\right)\right)^{\frac{1}{p}}\left(\left|f^{\prime}\left(\rho_{1}\right)\right|^{q}+3\left|f^{\prime}\left(\rho_{2}\right)\right|^{q}\right)^{\frac{1}{q}}\right\},
\end{array}
$$

where

$$
p=\frac{q}{q-1} \quad \text { and } \quad G_{p}\left(z_{1}, z_{2}\right):=\left|\int_{z_{1}}^{z_{*}}\right| x^{\omega}-\left.z_{1}^{\omega}\right|^{p} \mathrm{~d} x \mid
$$


Proof. By the convexity of $\left|f^{\prime}\right|^{q}$, we get (3.2), and thus, with the help of Hölder's inequality,

$$
\begin{aligned}
\left|\wp_{1}\right| & \leq \int_{\rho_{1}}^{\rho_{*}}\left(x^{\omega}-\rho_{1}^{\omega}\right)\left|f^{\prime}(x)\right| \mathrm{d} x \\
& \leq\left(\int_{\rho_{1}}^{\rho_{*}}\left(x^{\omega}-\rho_{1}^{\omega}\right)^{p} \mathrm{~d} x\right)^{\frac{1}{p}}\left(\int_{\rho_{1}}^{\rho_{*}}\left|f^{\prime}(x)\right|^{q} \mathrm{~d} x\right)^{\frac{1}{q}} \\
& =\left(G_{p}\left(\rho_{1}, \rho_{2}\right)\right)^{\frac{1}{p}}\left(\int_{\rho_{1}}^{\rho_{*}}\left|f^{\prime}(x)\right|^{q} \mathrm{~d} x\right)^{\frac{1}{q}} \\
& \leq\left(G_{p}\left(\rho_{1}, \rho_{2}\right)\right)^{\frac{1}{p}}\left(\left|f^{\prime}\left(\rho_{1}\right)\right|^{q} \int_{\rho_{1}}^{\rho_{*}} \frac{\rho_{2}-x}{\rho_{2}-\rho_{1}} \mathrm{~d} x+\left|f^{\prime}\left(\rho_{2}\right)\right|^{q} \int_{\rho_{1}}^{\rho_{*}} \frac{x-\rho_{1}}{\rho_{2}-\rho_{1}} \mathrm{~d} x\right)^{\frac{1}{q}} \\
& =\left(\frac{\rho_{2}-\rho_{1}}{8}\right)^{\frac{1}{q}}\left(G_{p}\left(\rho_{1}, \rho_{2}\right)\right)^{\frac{1}{p}}\left(3\left|f^{\prime}\left(\rho_{1}\right)\right|^{q}+\left|f^{\prime}\left(\rho_{2}\right)\right|^{q}\right)^{\frac{1}{q}} .
\end{aligned}
$$

Similarly,

$$
\begin{aligned}
\left|\wp_{2}\right| & \leq \int_{\rho_{*}}^{\rho_{2}}\left(\rho_{2}^{\omega}-x^{\omega}\right)\left|f^{\prime}(x)\right| \mathrm{d} x \\
& \leq\left(\int_{\rho_{*}}^{\rho_{2}}\left(\rho_{2}^{\omega}-x^{\omega}\right)^{p} \mathrm{~d} x\right)^{\frac{1}{p}}\left(\int_{\rho_{*}}^{\rho_{2}}\left|f^{\prime}(x)\right|^{q} \mathrm{~d} x\right)^{\frac{1}{q}} \\
& =\left(G_{p}\left(\rho_{2}, \rho_{1}\right)\right)^{\frac{1}{p}}\left(\int_{\rho_{*}}^{\rho_{2}}\left|f^{\prime}(x)\right|^{q} \mathrm{~d} x\right)^{\frac{1}{q}} \\
& \leq\left(G_{p}\left(\rho_{2}, \rho_{1}\right)\right)^{\frac{1}{p}}\left(\left|f^{\prime}\left(\rho_{1}\right)\right|^{q} \int_{\rho_{*}}^{\rho_{2}} \frac{\rho_{2}-x}{\rho_{2}-\rho_{1}} \mathrm{~d} x+\left|f^{\prime}\left(\rho_{2}\right)\right|^{q} \int_{\rho_{*}}^{\rho_{2}} \frac{x-\rho_{1}}{\rho_{2}-\rho_{1}} \mathrm{~d} x\right)^{\frac{1}{q}} \\
& =\left(\frac{\rho_{2}-\rho_{1}}{8}\right)^{\frac{1}{q}}\left(G_{p}\left(\rho_{2}, \rho_{1}\right)\right)^{\frac{1}{p}}\left(\left|f^{\prime}\left(\rho_{1}\right)\right|^{q}+3\left|f^{\prime}\left(\rho_{2}\right)\right|^{q}\right)^{\frac{1}{q}} .
\end{aligned}
$$

Employing now (2.9) completes the proof.

When we choose $\omega=1$ in Theorem 3.8, we get the following result.

Corollary 3.9. If $\left(H_{1}\right)$ holds and $\left|f^{\prime}\right|^{q}$ is convex for some $q>1$, and $p=q /(q-1)$, then

$$
\begin{aligned}
& \left|\frac{1}{\rho_{2}-\rho_{1}} \int_{\rho_{1}}^{\rho_{2}} f(x) \mathrm{d} x-f\left(\rho_{*}\right)\right| \\
& \quad \leq \frac{\rho_{2}-\rho_{1}}{16}\left(\frac{4}{p+1}\right)^{\frac{1}{p}}\left[\left(\left|f^{\prime}\left(\rho_{1}\right)\right|^{q}+3\left|f^{\prime}\left(\rho_{2}\right)\right|^{q}\right)^{\frac{1}{q}}+\left(3\left|f^{\prime}\left(\rho_{1}\right)\right|^{q}+\left|f^{\prime}\left(\rho_{2}\right)\right|^{q}\right)^{\frac{1}{q}}\right] .
\end{aligned}
$$

Proof. For $\omega=1$, we use (2.7) to calculate

$$
G_{p}\left(\rho_{1}, \rho_{2}\right)=\int_{\rho_{1}}^{\rho_{*}}\left(x-\rho_{1}\right)^{p} \mathrm{~d} x=\frac{\left(\rho_{*}-\rho_{1}\right)^{p+1}}{p+1}=\frac{\left(\rho_{2}-\rho_{1}\right)^{p+1}}{(p+1) 2^{p+1}}=G_{p}\left(\rho_{2}, \rho_{1}\right) .
$$

Noting $1 / p+1 / q=1$ and simplifying

$$
\frac{\left(\frac{\rho_{2}-\rho_{1}}{8}\right)^{\frac{1}{q}}\left(\frac{\left(\rho_{2}-\rho_{1}\right)^{p+1}}{(p+1) 2^{p+1}}\right)^{\frac{1}{p}}}{\rho_{2}-\rho_{1}}=\frac{\left(\rho_{2}-\rho_{1}\right) 4^{\frac{1}{p}}}{(p+1)^{\frac{1}{p}} 2^{\frac{1}{q}} 4^{\frac{1}{q}} 2^{1+\frac{1}{p}} 4^{\frac{1}{p}}}=\frac{\rho_{2}-\rho_{1}}{16}\left(\frac{4}{p+1}\right)^{\frac{1}{p}}
$$

and using Theorem 3.8 completes the proof.

Remark 3.10. Note that Corollary 3.9 was proved first in [13, Theorem 2.3]. Hence, our Theorem 3.8 is a generalization of [13, Theorem 2.3]. 
Table 4.1. $F\left(z_{1}, z_{2}\right)$

\begin{tabular}{|l||l|}
\hline$\omega$ & $\frac{8}{\left(z_{2}-z_{1}\right)^{2}} F\left(z_{1}, z_{2}\right)$ \\
\hline \hline 1 & 1 \\
2 & $\frac{1}{4}\left(5 z_{1}+3 z_{2}\right)$ \\
3 & $\frac{3}{4}\left(2 z_{1}^{2}+z_{1} z_{2}+z_{2}^{2}\right)$ \\
4 & $\frac{1}{24}\left(41 z_{1}^{3}+21 z_{1}^{2} z_{2}+15 z_{1} z_{2}^{2}+19 z_{2}^{3}\right)$ \\
5 & $\frac{5}{24}\left(9 z_{1}^{4}+5 z_{1}^{3} z_{2}+3 z_{1}^{2} z_{2}^{2}+3 z_{1} z_{2}^{3}+4 z_{2}^{4}\right)$ \\
6 & $\frac{1}{128}\left(257 z_{1}^{5}+155 z_{1}^{4} z_{2}+90 z_{1}^{3} z_{2}^{2}+70 z_{1}^{2} z_{2}+85 z_{1} z_{2}^{4}+111 z_{2}^{5}\right)$ \\
7 & $\frac{7}{384}\left(116 z_{1}^{6}+75 z_{1}^{5} z_{2}+45 z_{1}^{4} z_{2}^{2}+30 z_{1}^{3} z_{2} z_{2}^{3}+30 z_{1}^{2} z_{2}^{4}+39 z_{1} z_{2}^{5}+49 z_{2}^{6}\right)$ \\
\hline
\end{tabular}

Table 4.2. $F_{1}\left(z_{1}, z_{2}\right)$

\begin{tabular}{|l||l|}
\hline$\omega$ & $\frac{24}{\left(z_{2}-z_{1}\right)^{2}} F_{1}\left(z_{1}, z_{2}\right)$ \\
\hline \hline 1 & 1 \\
2 & $\frac{1}{8}\left(13 z_{1}+3 z_{2}\right)$ \\
3 & $\frac{3}{40}\left(27 z_{1}^{2}+11 z_{1} z_{2}+2 z_{2}^{2}\right)$ \\
4 & $\frac{1}{80}\left(183 z_{1}^{3}+99 z_{1}^{2} z_{2}+33 z_{1} z_{2}^{2}+5 z_{2}^{3}\right)$ \\
5 & $\frac{1}{112}\left(276 z_{1}^{4}+177 z_{1}^{3} z_{2}+81 z_{1}^{2} z_{2}^{2}+23 z_{1} z_{2}^{3}+3 z_{2}^{4}\right)$ \\
6 & $\frac{3}{1792}\left(1545 z_{1}^{5}+1107 z_{1}^{4} z_{2}+618 z_{1}^{3} z_{2}^{2}+246 z_{1}^{2} z_{2}+61 z_{1} z_{2}^{4}+7 z_{2}^{5}\right)$ \\
7 & $\frac{1}{768}\left(2053 z_{1}^{6}+1587 z_{1}^{5} z_{2}+1014 z_{1}^{4} z_{2}^{2}+502 z_{1}^{3} z_{2} z_{2}^{3}+177 z_{1}^{2} z_{2}^{4}+39 z_{1} z_{2}^{5}+4 z_{2}^{6}\right)$ \\
\hline
\end{tabular}

\section{Integer values of $\omega$}

Although the conformable integral mentioned in Section 1 is defined only for $\omega \in(0,1]$, the results presented in this paper hold for any $\omega>0$. By plugging the special values $\omega \in \mathbb{N}$, we can get some special cases of our presented inequalities. In Tables 4.1, 4.2, 4.3, and 4.4, we give the functions $F, F_{1}, F_{2}$, and $G$ for the values $\omega \in\{1,2,3,4,5,6,7\}$.

\section{Examples and applications}

In this final section, we give three distinct applications of our presented inequalities: First, inequalities involving the modified Bessel function of the first kind (for related inequalities, see [16, Section 4.3.1]). Second, inequalities involving the arithmetic mean and the $(\omega, r)$ th generalized logarithmic mean, and third, inequalities involving the midpoint formula (for related inequalities, see [12, Section 3]). 
Table 4.3. $F_{2}\left(z_{1}, z_{2}\right)$

\begin{tabular}{|l||l|}
\hline$\omega$ & $\frac{12}{\left(z_{2}-z_{1}\right)^{2}} F_{2}\left(z_{1}, z_{2}\right)$ \\
\hline \hline 1 & 1 \\
2 & $\frac{1}{16}\left(27 z_{1}+5 z_{2}\right)$ \\
3 & $\frac{3}{80}\left(58 z_{1}^{2}+19 z_{1} z_{2}+3 z_{2}^{2}\right)$ \\
4 & $\frac{1}{160}\left(405 z_{1}^{3}+177 z_{1}^{2} z_{2}+51 z_{1} z_{2}^{2}+7 z_{2}^{3}\right)$ \\
5 & $\frac{1}{224}\left(627 z_{1}^{4}+327 z_{1}^{3} z_{2}+129 z_{1}^{2} z_{2}^{2}+33 z_{1} z_{2}^{3}+4 z_{2}^{4}\right)$ \\
6 & $\frac{3}{3584}\left(3591 z_{1}^{5}+2109 z_{1}^{4} z_{2}+1014 z_{1}^{3} z_{2}^{2}+362 z_{1}^{2} z_{2}+83 z_{1} z_{2}^{4}+9 z_{2}^{5}\right)$ \\
7 & $\frac{1}{1536}\left(4868 z_{1}^{6}+3111 z_{1}^{5} z_{2}+1713 z_{1}^{4} z_{2}^{2}+758 z_{1}^{3} z_{2} z_{2}^{3}+246 z_{1}^{2} z_{2}^{4}+51 z_{1} z_{2}^{5}+5 z_{2}^{6}\right)$ \\
\hline
\end{tabular}

Table 4.4. $G\left(z_{1}, z_{2}\right)$

\begin{tabular}{|l||l|}
\hline$\omega$ & $\frac{8}{\left(z_{2}-z_{1}\right)^{2}} G\left(z_{1}, z_{2}\right)$ \\
\hline \hline 1 & 1 \\
2 & $\frac{1}{3}\left(5 z_{1}+z_{2}\right)$ \\
3 & $\frac{1}{8}\left(17 z_{1}^{2}+6 z_{1} z_{2}+z_{2}^{2}\right)$ \\
4 & $\frac{1}{20}\left(49 z_{1}^{3}+23 z_{1}^{2} z_{2}+7 z_{1} z_{2}^{2}+z_{2}^{3}\right)$ \\
5 & $\frac{1}{48}\left(129 z_{1}^{4}+72 z_{1}^{3} z_{2}+30 z_{1}^{2} z_{2}^{2}+8 z_{1} z_{2}^{3}+z_{2}^{4}\right)$ \\
6 & $\frac{1}{112}\left(321 z_{1}^{5}+201 z_{1}^{4} z_{2}+102 z_{1}^{3} z_{2}^{2}+38 z_{1}^{2} z_{2}+9 z_{1} z_{2}^{4}+z_{2}^{5}\right)$ \\
7 & $\frac{1}{256}\left(769 z_{1}^{6}+522 z_{1}^{5} z_{2}+303 z_{1}^{4} z_{2}^{2}+140 z_{1}^{3} z_{2} z_{2}^{3}+47 z_{1}^{2} z_{2}^{4}+10 z_{1} z_{2}^{5}+z_{2}^{6}\right)$ \\
\hline
\end{tabular}

\subsection{Bessel functions}

Consider the function $\mathcal{B}_{\varrho}:(0, \infty) \rightarrow[1, \infty)$ defined by

$$
\mathcal{B}_{\varrho}(x)=2^{\varrho} \Gamma(\varrho+1) x^{-\varrho} \mathcal{B}_{\varrho}^{1}(x),
$$

where $\mathcal{B}_{\varrho}^{1}$ is the modified Bessel function of the first kind defined by (see [27, (2) on page 77])

$$
\mathcal{B}_{\varrho}^{1}(x)=\sum_{n=0}^{\infty} \frac{\left(\frac{x}{2}\right)^{\varrho+2 n}}{n ! \Gamma(\varrho+1+n)}, \quad x \in \mathbb{R} .
$$

In $[27,(6)$ on page 79$]$, the first derivative of $\mathcal{B}_{\varrho}$ is given by

$$
\mathcal{B}_{\varrho}^{\prime}(x)=\frac{x \mathcal{B}_{\varrho+1}(x)}{2(\varrho+1)},
$$


and the second derivative can easily be calculated from (5.1) to be

$$
\mathcal{B}_{\varrho}^{\prime \prime}(x)=\frac{x^{2} \mathcal{B}_{\varrho+2}(x)}{4(\varrho+1)(\varrho+2)}+\frac{\mathcal{B}_{\varrho+1}(x)}{2(\varrho+1)} .
$$

Example 5.1. Let $0<\rho_{1}<\rho_{2}$ and $\varrho>-1$. Then, by applying Corollary 3.2 with $f=\mathcal{B}_{\varrho}^{\prime}$ (note that all assumptions are satisfied) and the identities (5.1) and (5.2), we have

$$
\begin{aligned}
\mid \frac{\mathcal{B}_{\varrho}\left(\rho_{2}\right)-\mathcal{B}_{\varrho}\left(\rho_{1}\right)}{\rho_{2}-\rho_{1}} & -\frac{\rho_{1}+\rho_{2}}{4(\varrho+1)} \mathcal{B}_{\varrho+1}\left(\frac{\rho_{1}+\rho_{2}}{2}\right) \mid \\
& \leq \frac{\rho_{2}-\rho_{1}}{16}\left[\frac{\rho_{1}^{2} \mathcal{B}_{\varrho+2}\left(\rho_{1}\right)+\rho_{2}^{2} \mathcal{B}_{\varrho+2}\left(\rho_{2}\right)}{2(\varrho+1)(\varrho+2)}+\frac{\mathcal{B}_{\varrho+1}\left(\rho_{1}\right)+\mathcal{B}_{\varrho+1}\left(\rho_{2}\right)}{\varrho+1}\right] .
\end{aligned}
$$

Example 5.2. Let $0<\rho_{1}<\rho_{2}, \varrho>-1$, and $q \geq 1$. Then, by applying Corollary 3.6 with $f=\mathcal{B}_{\varrho}^{\prime}$ (note that all assumptions are satisfied) and the identities (5.1) and (5.2), we get

$$
\begin{aligned}
&\left|\frac{\mathcal{B}_{\varrho}\left(\rho_{2}\right)-\mathcal{B}_{\varrho}\left(\rho_{1}\right)}{\rho_{2}-\rho_{1}}-\frac{\rho_{1}+\rho_{2}}{4(\varrho+1)} \mathcal{B}_{\varrho+1}\left(\frac{\rho_{1}+\rho_{2}}{2}\right)\right| \\
& \leq \frac{\rho_{2}-\rho_{1}}{16(\varrho+1) 3^{\frac{1}{q}}}\left\{\left[2\left(\frac{\rho_{1}^{2} \mathcal{B}_{\varrho+2}\left(\rho_{1}\right)}{2(\varrho+2)}+\mathcal{B}_{\varrho+1}\left(\rho_{1}\right)\right)^{q}+\left(\frac{\rho_{2}^{2} \mathcal{B}_{\varrho+2}\left(\rho_{2}\right)}{2(\varrho+2)}+\mathcal{B}_{\varrho+1}\left(\rho_{2}\right)\right)^{q}\right]^{\frac{1}{q}}\right. \\
&\left.+\left[\left(\frac{\rho_{1}^{2} \mathcal{B}_{\varrho+2}\left(\rho_{1}\right)}{2(\varrho+2)}+\mathcal{B}_{\varrho+1}\left(\rho_{1}\right)\right)^{q}+2\left(\frac{\rho_{2}^{2} \mathcal{B}_{\varrho+2}\left(\rho_{2}\right)}{2(\varrho+2)}+\mathcal{B}_{\varrho+1}\left(\rho_{2}\right)\right)^{q}\right]^{\frac{1}{q}}\right\} .
\end{aligned}
$$

Example 5.3. Let $0<\rho_{1}<\rho_{2}, \varrho>-1, q>1$, and $p=q /(q-1)$. Then, by applying Corollary 3.9 with $f=\mathcal{B}_{\varrho}^{\prime}$ (note that all assumptions are satisfied) and the identities (5.1) and (5.2), we obtain

$$
\begin{aligned}
& \left|\frac{\mathcal{B}_{\varrho}\left(\rho_{2}\right)-\mathcal{B}_{\varrho}\left(\rho_{1}\right)}{\rho_{2}-\rho_{1}}-\frac{\rho_{1}+\rho_{2}}{4(\varrho+1)} \mathcal{B}_{\varrho+1}\left(\frac{\rho_{1}+\rho_{2}}{2}\right)\right| \\
& \leq \frac{\rho_{2}-\rho_{1}}{32(\varrho+1)} \sqrt[p]{\frac{4}{\varrho+1}}\left\{\left[\left(\frac{\rho_{1}^{2} \mathcal{B}_{\varrho+2}\left(\rho_{1}\right)}{2(\varrho+2)}+\mathcal{B}_{\varrho+1}\left(\rho_{1}\right)\right)^{q}+3\left(\frac{\rho_{2}^{2} \mathcal{B}_{\varrho+2}\left(\rho_{2}\right)}{2(\varrho+2)}+\mathcal{B}_{\varrho+1}\left(\rho_{2}\right)\right)^{q}\right]^{\frac{1}{q}}\right. \\
& \left.+\left[3\left(\frac{\rho_{1}^{2} \mathcal{B}_{\varrho+2}\left(\rho_{1}\right)}{2(\varrho+2)}+\mathcal{B}_{\varrho+1}\left(\rho_{1}\right)\right)^{q}+\left(\frac{\rho_{2}^{2} \mathcal{B}_{\varrho+2}\left(\rho_{2}\right)}{2(\varrho+2)}+\mathcal{B}_{\varrho+1}\left(\rho_{2}\right)\right)^{q}\right]^{\frac{1}{q}}\right\} .
\end{aligned}
$$

\subsection{Special means}

Let $r \in \mathbb{R} \backslash\{-\omega, 0\}, \omega \in(0,1]$, and $\rho_{1}, \rho_{2} \in \mathbb{R}$ with $0<\rho_{1}<\rho_{2}$. Then we consider the arithmetic mean and the $(\omega, r)$ th generalized logarithmic mean defined by

$$
A\left(\rho_{1}, \rho_{2}\right)=\frac{\rho_{1}+\rho_{2}}{2} \quad \text { and } \quad L_{(\omega, r)}\left(\rho_{1}, \rho_{2}\right)=\left[\frac{\omega\left(\rho_{2}^{r+\omega}-\rho_{1}^{r+\omega}\right)}{(r+\omega)\left(\rho_{2}^{\omega}-\rho_{1}^{\omega}\right)}\right]^{\frac{1}{r}} .
$$

Example 5.4. Let $r>1$ and $f(x)=x^{r}$ for all $x>0$. It is easy to see that $\left|f^{\prime}\right|$ is convex on $\left[\rho_{1}, \rho_{2}\right]$. Then, by applying Theorem 3.1, the $r$ th powers of the two means in (5.4) differ from each other at most by

$$
\frac{r}{\rho_{2}^{\omega}-\rho_{1}^{\omega}}\left(\rho_{1}^{r-1} F\left(\rho_{1}, \rho_{2}\right)+\rho_{2}^{r-1} F\left(\rho_{2}, \rho_{1}\right)\right),
$$

where $F$ is given in Theorem 3.1 . 
Example 5.5. Let $q \geq 1, r>1$, and $f(x)=x^{r}$ for all $x>0$. Then, by applying Theorem 3.4 , the $r$ th powers of the two means in (5.4) differ from each other at most by

$$
\begin{aligned}
& \frac{r}{\rho_{2}^{\omega}-\rho_{1}^{\omega}}\{\left(G\left(\rho_{1}, \rho_{2}\right)\right)^{1-\frac{1}{q}}\left(\rho_{1}^{q(r-1)} F_{2}\left(\rho_{1}, \rho_{2}\right)+\rho_{2}^{q(r-1)} F_{1}\left(\rho_{1}, \rho_{2}\right)\right)^{\frac{1}{q}} \\
&\left.+\left(G\left(\rho_{2}, \rho_{1}\right)\right)^{1-\frac{1}{q}}\left(\rho_{1}^{q(r-1)} F_{1}\left(\rho_{2}, \rho_{1}\right)+\rho_{2}^{q(r-1)} F_{2}\left(\rho_{2}, \rho_{1}\right)\right)^{\frac{1}{q}}\right\},
\end{aligned}
$$

where $F_{1}, F_{2}$, and $G$ are given in Lemma 2.1.

Example 5.6. Let $q>1, r>1$, and $f(x)=x^{r}$ for all $x>0$. Then, by applying Theorem 3.8 , the $r$ th powers of the two means in (5.4) differ from each other at most by

$$
\begin{gathered}
\frac{r}{\rho_{2}^{\omega}-\rho_{1}^{\omega}} \sqrt[p]{\frac{\rho_{2}-\rho_{1}}{8}}\left\{\left(G_{p}\left(\rho_{1}, \rho_{2}\right)\right)^{\frac{1}{p}}\left(\rho_{1}^{q(r-1)}+3 \rho_{2}^{q(r-1)}\right)^{\frac{1}{q}}\right. \\
\left.+\left(G_{p}\left(\rho_{2}, \rho_{1}\right)\right)^{\frac{1}{p}}\left(3 \rho_{1}^{q(r-1)}+\rho_{2}^{q(r-1)}\right)^{\frac{1}{q}}\right\},
\end{gathered}
$$

where $p=q /(q-1)$ and $G_{p}$ is given in Theorem 3.8.

\subsection{Midpoint approximations}

Let $\mathcal{P}$ be the partition $\rho_{1}=x_{0}<x_{1}<\ldots<x_{n}=\rho_{2}$ of the interval $\left[\rho_{1}, \rho_{2}\right]$. We consider the quadrature formula

$$
\int_{\rho_{1}}^{\rho_{2}} f(x) \mathrm{d}_{\omega} x=M_{\omega}(f, \mathcal{P})+E_{\omega}(f, \mathcal{P})
$$

where

$$
M_{\omega}(f, \mathcal{P})=\sum_{i=0}^{n-1} f\left(\frac{x_{i}+x_{i+1}}{2}\right) \frac{x_{i+1}^{\omega}-x_{i}^{\omega}}{\omega}
$$

is the midpoint approximation and $E_{\omega}(f, \mathcal{P})$ denotes the corresponding approximation error.

Example 5.7. If $\left(\mathrm{H}_{\omega}\right)$ holds and $\left|f^{\prime}\right|$ is convex, then, by applying Theorem 3.1 on the subintervals $\left[x_{i}, x_{i+1}\right], i=0, \ldots, n-1$, of the partition $\mathcal{P}$, we have

$$
\begin{aligned}
\left|f\left(\frac{x_{i}+x_{i+1}}{2}\right)-\frac{\omega}{x_{i+1}^{\omega}-x_{i}^{\omega}} \int_{x_{i}}^{x_{i+1}} f(x) \mathrm{d}_{\omega} x\right| & \\
\leq & \leq \frac{F\left(x_{i}, x_{i+1}\right)\left|f^{\prime}\left(x_{i}\right)\right|+F\left(x_{i+1}, x_{i}\right)\left|f^{\prime}\left(x_{i+1}\right)\right|}{x_{i+1}^{\omega}-x_{i}^{\omega}},
\end{aligned}
$$

where $F$ is given in Theorem 3.1, and then by adding, we get

$$
\left|E_{\omega}(f, \mathcal{P})\right| \leq \frac{1}{\omega} \sum_{i=0}^{n-1}\left[F\left(x_{i}, x_{i+1}\right)\left|f^{\prime}\left(x_{i}\right)\right|+F\left(x_{i+1}, x_{i}\right)\left|f^{\prime}\left(x_{i+1}\right)\right|\right] .
$$

Example 5.8. If $\left(\mathrm{H}_{\omega}\right)$ holds and $\left|f^{\prime}\right|^{q}$ is convex for some $q \geq 1$, then, by applying Theorem 3.4 on the subintervals $\left[x_{i}, x_{i+1}\right], i=0, \ldots, n-1$, of the partition $\mathcal{P}$, and then adding, we have

$$
\begin{aligned}
\left|E_{\omega}(f, \mathcal{P})\right| \leq \frac{1}{\omega} \sum_{i=0}^{n-1} & \left\{\left(G\left(x_{i}, x_{i+1}\right)\right)^{1-\frac{1}{q}}\left(F_{2}\left(x_{i}, x_{i+1}\right)\left|f^{\prime}\left(x_{i}\right)\right|^{q}+F_{1}\left(x_{i}, x_{i+1}\right)\left|f^{\prime}\left(x_{i+1}\right)\right|^{q}\right)^{\frac{1}{q}}\right. \\
& \left.+\left(G\left(x_{i+1}, x_{i}\right)\right)^{1-\frac{1}{q}}\left[F_{1}\left(x_{i+1}, x_{i}\right)\left|f^{\prime}\left(x_{i}\right)\right|^{q}+F_{2}\left(x_{i+1}, x_{i}\right)\left|f^{\prime}\left(x_{i+1}\right)\right|^{q}\right]^{\frac{1}{q}}\right\},
\end{aligned}
$$

where $F_{1}, F_{2}$, and $G$ are given in Lemma 2.1. 
Example 5.9. If $\left(\mathrm{H}_{\omega}\right)$ holds and $\left|f^{\prime}\right|^{q}$ is convex for some $q>1$, then, by applying Theorem 3.8 on the subintervals $\left[x_{i}, x_{i+1}\right], i=0, \ldots, n-1$, of the partition $\mathcal{P}$, and then adding, we obtain

$$
\begin{array}{r}
\left|E_{\omega}(f, \mathcal{P})\right| \leq \frac{1}{\omega \sqrt[q]{8}} \sum_{i=0}^{n-1} \sqrt[q]{x_{i+1}-x_{i}}\left\{\left(G_{p}\left(x_{i}, x_{i+1}\right)\right)^{\frac{1}{p}}\left[\left|f^{\prime}\left(x_{i}\right)\right|^{q}+3\left|f^{\prime}\left(x_{i+1}\right)\right|^{q}\right]^{\frac{1}{q}}\right. \\
\left.+\left(G_{p}\left(x_{i+1}, x_{i}\right)\right)^{\frac{1}{p}}\left[3\left|f^{\prime}\left(x_{i}\right)\right|^{q}+\left|f^{\prime}\left(x_{i+1}\right)\right|^{q}\right]^{\frac{1}{q}}\right\}
\end{array}
$$

where $p=q /(q-1)$ and $G_{p}$ is given in Theorem 3.8.

\section{References}

[1] T. Abdeljawad, On conformable fractional calculus, J. Comput. Appl. Math. 279, 57-66, 2015.

[2] P. Agarwal, S.S. Dragomir, M. Jleli and B. Samet, Advances in mathematical inequalities and applications, Trends in Mathematics. Birkhäuser/Springer, Singapore, 2018.

[3] F.M. Atıcı and H. Yaldı, Convex functions on discrete time domains, Canad. Math. Bull. 59 (2), 225-233, 2016.

[4] J. Barić, R. Bibi, M. Bohner, A. Nosheen and J. Pečarić, Jensen inequalities on time scales, volume 9 of Monographs in Inequalities, ELEMENT, Zagreb, 2015.

[5] J. Barić, R. Bibi, M. Bohner and J. Pečarić, Time scales integral inequalities for superquadratic functions, J. Korean Math. Soc. 50 (3), 465-477, 2013.

[6] K.S. Chiu and T. Li, Oscillatory and periodic solutions of differential equations with piecewise constant generalized mixed arguments, Math. Nachr. 292 (10), 2153-2164, 2019.

[7] P. Ciatti, M.G. Cowling and F. Ricci, Hardy and uncertainty inequalities on stratified Lie groups, Adv. Math. 277, 365-387, 2015.

[8] H. Gunawan and Eridani, Fractional integrals and generalized Olsen inequalities, Kyungpook Math. J. 49 (1), 31-39, 2009.

[9] M.A. Hanson, On sufficiency of the Kuhn-Tucker conditions, J. Math. Anal. Appl. 80 (2), 545-550, 1981.

[10] M. Iqbal, M.I. Bhatti and K. Nazeer, Generalization of inequalities analogous to Hermite-Hadamard inequality via fractional integrals, Bull. Korean Math. Soc. 52 (3), 707-716, 2015.

[11] H. Kalsoom, M.A. Ali, M. Idrees, P. Agarwal and M. Arif, New post quantum analogues of Hermite-Hadamard type inequalities for interval-valued convex functions, Math. Probl. Eng. Art. ID 5529650, 17 pages, 2021.

[12] M.A. Khan, T. Ali, S.S. Dragomir and M.Z. Sarikaya, Hermite-Hadamard type inequalities for conformable fractional integrals, Rev. R. Acad. Cienc. Exactas Fís. Nat. Ser. A Mat. RACSAM 112 (4),1033-1048, 2018.

[13] U.S. Kirmaci, Inequalities for differentiable mappings and applications to special means of real numbers and to midpoint formula, Appl. Math. Comput. 147 (1), 137146, 2004.

[14] T. Li, N. Pintus and G. Viglialoro, Properties of solutions to porous medium problems with different sources and boundary conditions, Z. Angew. Math. Phys. 70 (3), No. 86, 18, 2019.

[15] T. Li and G. Viglialoro, Boundedness for a nonlocal reaction chemotaxis model even in the attraction-dominated regime, Differential Integral Equations, 34 (5-6), 315-336, 2021.

[16] K. Mehrez and P. Agarwal, New Hermite-Hadamard type integral inequalities for convex functions and their applications, J. Comput. Appl. Math. 350, 274-285, 2019. 
[17] P.O. Mohammed, Hermite-Hadamard inequalities for Riemann-Liouville fractional integrals of a convex function with respect to a monotone function, Math. Methods Appl. Sci. 44 (3), 2314-2324, 2021.

[18] P.O. Mohammed and T. Abdeljawad, Modification of certain fractional integral inequalities for convex functions, Adv. Difference Equ. 2020, 69, 2020.

[19] P.O. Mohammed, T. Abdeljawad, D. Baleanu, A. Kashuri, F. Hamasalh and P. Agarwal, New fractional inequalities of Hermite-Hadamard type involving the incomplete gamma functions, J. Inequal. Appl. 263, 1-16, 2020.

[20] P.O. Mohammed and F.K. Hamasalh, New conformable fractional integral inequalities of Hermite-Hadamard type for convex functions, Symmetry, 11 (2), 2019.

[21] P.O. Mohammed and M.Z. Sarikaya, Hermite-Hadamard type inequalities for Fconvex function involving fractional integrals, J. Inequal. Appl. 359, 1-33, 2018.

[22] P.O. Mohammed and M.Z. Sarikaya, On generalized fractional integral inequalities for twice differentiable convex functions, J. Comput. Appl. Math. 372, 112740, 15, 2020.

[23] P.O. Mohammed, M.Z. Sarikaya and D. Baleanu, On the generalized HermiteHadamard inequalities via the tempered fractional integrals, Symmetry 12 (4), 2020.

[24] F. Qi, P.O. Mohammed, J.C. Yao and Y.H. Yao, Generalized fractional integral inequalities of Hermite-Hadamard type for $(\alpha, m)$-convex functions, J. Inequal. Appl. 135, 1-17, 2019.

[25] M. Ruzhansky, Y.J. Cho, P. Agarwal and I. Area, eds, Advances in real and complex analysis with applications, Trends in Mathematics. Birkhäuser/Springer, Singapore, 2017.

[26] M.Z. Sarikaya and H. Yildirim, On Hermite-Hadamard type inequalities for RiemannLiouville fractional integrals, Miskolc Math. Notes, 17 (2), 1049-1059, 2016.

[27] G.N. Watson, A treatise on the theory of Bessel functions, Cambridge Mathematical Library. Cambridge University Press, Cambridge, 1995.

[28] H. Yaldız and P. Agarwal, s-convex functions on discrete time domains, Analysis, 37 (4), 179-184, 2017.

[29] X.X. You, M.A. Ali, H. Budak, P. Agarwal and Y.M. Chu, Extensions of HermiteHadamard inequalities for harmonically convex functions via generalized fractional integrals, J. Inequal. Appl. 102, 1-22, 2021. 